\title{
Agent-Based Modeling of Supply Chains in Critical Situations
}

\author{
Jarosław Koźlak, Grzegorz Dobrowolski, and Edward Nawarecki \\ Institute of Computer Science \\ AGH University of Science and Technology, Kraków, Poland \\ $\{$ kozlak, grzela, nawar\}eagh.edu.pl
}

\begin{abstract}
Supply chains are discussed in this article. They are viewed as intelligent decentralized systems that meet the agent paradigm. Thus possibility of arising critical situations in the chains can be analyzed in the agent-based manner. The work is focused on applying an overall methodology dedicated to the discovery of crises and support of anti-crisis activities. The agent-based model is proposed that incorporates majority of features of supply chains so that the modeling of crises can be wide-ranging and easy. As an illustration some simulation results are presented.
\end{abstract}

\section{Introduction}

Intelligent decentralized systems that meet the agent paradigm [2] can be of two types: designed from scratch as multi-agent ones (operating in the virtual world, e.g. network information services, virtual enterprises) and existing in the reality as a set of cooperating autonomous subsystems of whatever origin (e.g. transportation systems, industrial complexes) that can be analyzed in agent-based manner.

Such systems are marked by possibility of arising critical situations that can be caused by both outer (e.g. undesirable interference or the forces of nature) and inner (e.g. resource deficit, local damages) factors. Such is the source of an idea to embed considerations about crises in the field of multi-agent systems - MAS [3]6].

As a consequence, in the real case an assumption must be taken that the system under consideration has agent-based conceptualization that can serve as a means for analysis of its operation, especially in critical situations. If the description occurs to be enough precise, a simulation (computerized) model of its behavior can be built. Results of the simulation studies would be the scenarios of crises progress. Investigation of the scenarios would lead to finding a strategy of avoiding the particular crisis or, at least, reducing its effects [96].

Some difficulties of crisis identification, evaluation of possible effects and prevention (anti-crisis) actions come from general features of multi-agent systems (autonomy of the agent's decisions, lack of global information) as well as their dynamics (consequences appear after an operation in unpredictable manner).

A set of enterprises which activities can be characterized as production or providing services, distribution or trading of some raw materials, by-products or market goods, called a supply chain, constitute a system that can be numbered among the discussed 
above and the ideas of agent and multi-agent system can be useful to solve managerial problems dealing with it.

The same approach is present in research reported by some number of publications, among which it is worth to mention here at least [7/4/5]8]. The first one contains a comparative study of the agent-based approach versus some others proposed earlier. Searching for the answer to question whether agents that construct supply chains can do it better than human decision makers, especially with respect to changing market conditions is a subject of [4]. In [5] a reader can find a limited overview of the discussed field oriented to comparison of different applications of multi-agent systems to modeling and managing the chains. As bases of reported algorithms the following are mentioned: evolutionary algorithms, special languages (grammars), various auction (Contract Net) and negotiation schemas.

The paper is organized as follows. The initial part describes the proposed model of a critical situation with discussion of its most important aspects. Some assumptions and architecture solutions of how to manage with the defined critical situations is proposed also. The next section 3 introduces an area of application which is management of supply chains. Section 4 gives description of an agent-based model that, following the main methodology assumption, is prepared to carry out simulation studies of crises in the chains. The chosen results of simulation studies illustrating the approach are presented at the end.

\section{Critical Situations in MAS}

A critical situation is recognized as a particular state or sequence of states that violate or lead to violation of the global as well as local (the agents') goals of a system. Thus critical situations can be local (concerning a single agent) and global (involving not only all but also a group of agents). Arising of a local crisis may entail a global one in the future, but functional abilities of a system very often allow avoiding the consequences at the global level. Such phenomenon straight results from the basic features of multi-agent systems. One may say that some anti-crisis mechanisms (in the above sense) are already incorporated. On the contrary, threat of a global crisis usually requires especially invented mechanisms.

A crisis among a group of agents is treated here as a global one because of similar way of the state description and the obvious fact that such crisis must emerge with respect to a partial or side- goal of a system.

The above characteristics allow to define general conditions of management of critical situations:

- possibility of observation (monitoring) of the system state based on observation of the agents' states individually,

- adoption of the adequate ways of evaluation of a state in order to achieve operational criteria of critical situations recognition,

- availability of appropriate anti-crisis mechanisms.

Degree of realization of the above postulates can be regarded as a determinant of the system immunity against a crisis. As it has been signalled, a flexible by nature 
multi-agent system has some elements of the mechanisms implemented either as parts of the agent's algorithms or in a way of communication or organization of a system (or a sub-system).

Let us discuss the conditions firstly for the case of local critical situations. In the obvious way an agent monitors his state as well as evaluates it on his own. In each state he determines a set of admissible actions. Significant reduction of the set can be an indication of the crisis. If the agent must consider actions like "do nothing" or selfdestruction, it is not only the indication but a kind of remedy also.

Although application of the agent's strategy is oriented towards a decision, it is also the evaluation of the state. If some ranking of the actions is prepared according to the utility coefficients, its values can be used for formulation of a crisis criterion. Then decline in utility can be regarded as a sign of a crisis. Finally, if the both mechanisms occur to be insufficient, the applied choice function can be augmented with an element intended for monitoring of crises and causing activation of built-in especially anti-crisis actions.

The similar analysis with respect to global critical situations is a bit harder. This is because of a problem with determining the state of a multi-agent system. The state can be easily defined as composition of the agent's states but its calculation is usually operationally impossible. Such situation comes from the following features of a system.

- There are no strong enough synchronization mechanism to determine simultaneity of the agent's states.

- The system state is highly multi-dimensional so that the high cost of information acquisition should be taken into account.

- The agents are autonomous. They usually intend to disclose only as much information as it is necessary for the system operation.

In the general case it is assumed that agent reveals just a sub-space of his state or some evaluation of its state. Restriction of the state is accepted as a report while the evaluation is regarded as subjective. Of course, interpretation of the above information is known around the system. It is worthwhile to mention here that a state comprises also information about history so that the evaluation can have dynamic character.

Putting all descriptions of the agent's state together possibly in a single place and regarding them as simultaneous is the only way to construct a description of the state of the whole system. The evaluation - similarly as in the case of a single agent can be oriented towards critical situation. Adoption of a special shape of the evaluation functions and the appropriate definition of subsets of their values opens possibility of specialized tracking of the system states. For example, the values can be done by linguistic values: normal, preferred, danger, crisis. In its simplest form tracking can be just memorization of monitoring data and introduction of them into an evaluation procedure.

Following the pointed earlier ways of definition of the global goal, two kinds of critical situations can be introduced: direct and indirect. The direct means the threat of loosing operability of the system in consequence of unavailability of the some agents' actions. The primary cause of an indirect critical situation is a lack of resources (violation of the appropriate balance) that, in turn, gives deficit of functionality. 


\section{Structure of Agent-Based Management of Crises}

The previous section is the point of departure towards the idea of the structure and functionality of a system devoted to management of critical situations. The proposed solution is aimed to be universal. It is also lack of details that strongly depend on a particular considered system exposed to crises. Before such details for the case of supply

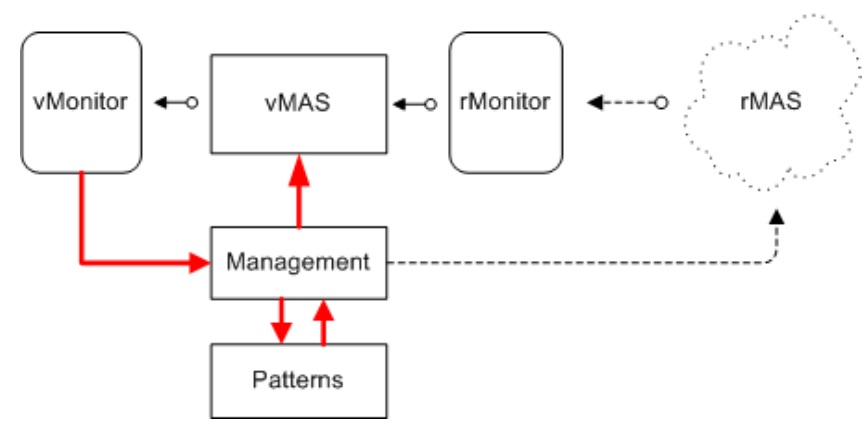

Fig. 1. General Structure of Management of Critical Situations

chains will be presented, let us analyzed generals on figure 1 The main elements of the structure are:

rMAS an agent-based conceptualization of real systems of some kind; there can be pointed out also the concrete real system accords with this conceptualization;

vMAS an agent-based computerized model of the conceptualization thus systems of that kind and finally the concrete one.

The presented structure is a direct consequence of a methodological rule, that creates a basis of majority of research using modeling and simulation. The rule can be formulated here as follows. Pairs of mechanisms respectively of rMAS and vMAS are considered. For each pair two statements are true: a real mechanism is adequately modeled or the virtual one is adequately and effectively implemented in the reality. It must be pointed out that the formulation not only comprises requirements of adequate modeling but indicates possibility of searching for such mechanisms basing on the model (vMAS) and applying them in the reality (rMAS) as well.

Appropriate extraction and flows of data in the described structure are done by two monitoring modules, respectively: rMonitor and vMonitor. The former serves as a provider of available information about the real system (rMAS), the latter is design mainly to aggregate generated output data of the model (vMAS) which can be regarded as the standard computer science problem. Of course, the amount of the data dictates application of proper techniques.

As it is assumed, the invented mechanisms are to be implemented. It is represented by block Management in the general structure that plays, in fact, a three-fold role. 
1. Working out mechanisms of crisis recognition (patterns) or anti-crisis policy which are to be implemented in rMAS at the local level of a single agent.

2. Working out global mechanisms of crisis recognition or anti-crisis policy.

3. Implementation of the global mechanisms or on-line management using previously obtained patterns.

Element Patterns represents all effects of Management functionality, including patterns applied in the on-line management.

\section{Agent-Based Management of Supply Chains}

A set of enterprises whose activities can be characterized as production or providing services, distribution or trading of some raw materials, by-products or market goods is regarded here as a supply chain. Moreover, it is assumed that the enterprises can cooperate with each other and are (or can) be managed as a whole but only in the sense of some decentralized decision mechanisms.

Such description indicates a fact that the supply chain constitutes a complex system and in that case on one hand needs very special methods of management, on the other hand is fragile in the perspective of various types of crises. Management of the supply chain must take into account first and foremost that the system elements are highly autonomous and keep information about themselves strictly confidential. Thus managerial methods applied must be of the decentralized kind as well as based on market mechanisms and legal agreements.

The model (vMAS) consists of two types of agents - enterprise agents and client agents. As additional elements commodities which are produced or processed by the enterprises are modeled also. Enterprise agents have production lines at their disposal that realize transformation of commodities. Agents of both types are described by three groups of parameters: configuration parameters of the lines (e.g. capacity, production cost, maintenance cost, quality of production), decision parameters that determine behavior of the agent together with other parameters describing the agent that can be modified by him (e.g. margin value, several weight coefficient 1 ), and finally, parameters that describe the agent also but change as an indirect consequence of activities of other agents (e.g. financing, prestige on a market).

The general rule of the model is as follows. In an assumed time regime clientagents place orders for chosen final products. Enterprise-agents that are able to fulfill some of those orders try to construct adequate supply chains. Usually there are several enterprise-agents engaged in a single chain. Finally as a result of several auctions the whole system can be organized and the orders done.

The model assumes a set of parameters that describe a production line. The parameters depend on each other according to the following equation.

$$
k=\frac{a q}{b c} d w
$$

where: $k$ - maintenance cost of a line, $q$ - quality of production, $c$ - production cost, $w$ - capacity, $a, b, c$ - weight coefficients of parameters $q, c, w$ respectively.

\footnotetext{
${ }^{1}$ The coefficients are presented in the course of the article.
} 
Interchange of goods is modeled with a kind of markets. Entering the markets, suppliers and customers evaluate interesting sale and purchase offers. Formulas which are used in the model can be sketched out as in eq. (2) - evaluation of a purchase offer and eq. (3) - evaluation of a sale one.

$$
o_{b}=o_{b}(p, p w, n, n w, t, t w)
$$

where: $p$ - representation of the customer prestige, $t$ - delivery time needed, $n$ - quantity, $p w, t w, n w-$ weight coefficients of parameters $p, t, n$ respectively.

$$
o_{s}=o_{s}(p, n, q, t, c, p w, q w, t w, c w)
$$

where: $p$-supplier prestige, $t$ - offered delivery time, $n$ - quantity, $q$ - offered quality, $c$ - price, $p w, t w, q w, c w$ - weight coefficients of parameters $p, t, q, c$.

Next the best offers are chosen and a kind of agreement set. It is assumed that all the agreements are realized in turn. The decision (auction) algorithm is the same for the whole model but differs in parameters of each enterprise-agent. Moreover, there is a mechanism which realize optimal tuning of the parameters in the course of simulation. The mechanism models possibility of forecasting studies by a real enterprise. It is done based on the idea of evolutionary computation.

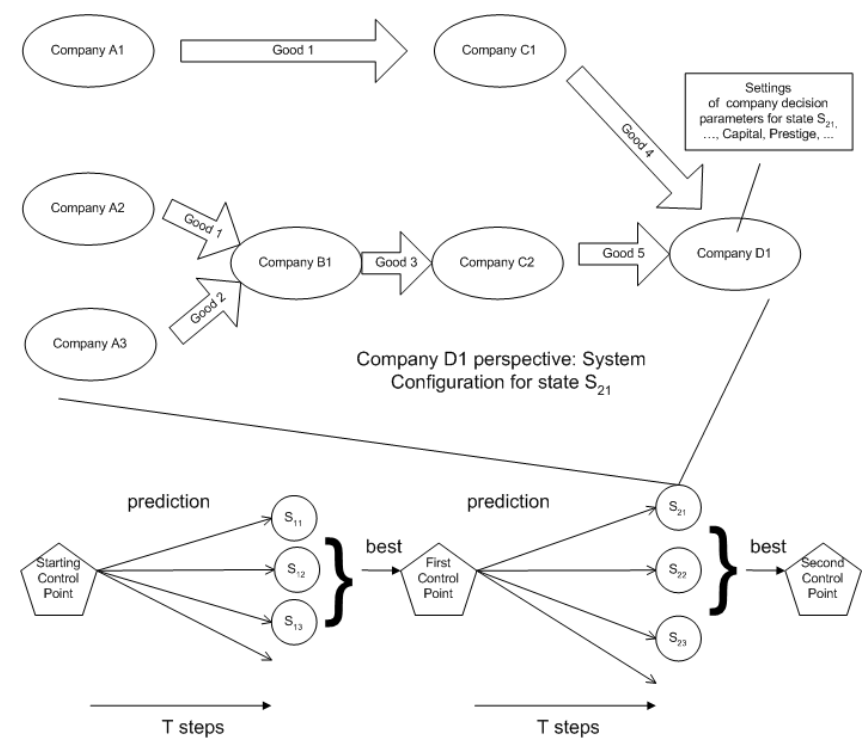

Fig. 2. Simulation model

Whenever an enterprise-agent faces a decision, the main stream of simulation is suspended and the predicting mechanism started (see fig. 2). At the beginning a population of enterprise-agents is generated as pseudo-random variation (mutation) of the agent's parameters which represents the enterprise under consideration. A number of 
sub-simulations with a given (predicting) horizon are carried out for each agent of the population in turn. Next each such a variant is evaluated on the basis of the value of enterprise capital gained and the best chosen. Then the main stream is resumed and the best agent can work. Assumed in the model, an expression that represents the capital of an enterprise is as follows.

$$
u=f\left(l, c_{o}, g_{b}\right)+g(e)
$$

where: $f$ - function that describes changes of the capital, $l$ - maintenance cost that depends on the parameters of a line, $c_{o}$ - total cost of raw materials, $g_{b}$ - profit gained as a result of the winning auction, $g(e)$ - profit that is gained.

The implementation of the model [1] is an application of medium degree of complication. It has layered software architecture. The following layers can be shown to give the general overwiev of it: Java Virtual Machine, JADE - Java Agent DEvelopment Platform that conforms to FIPA - Foundation for Intelligent Physical Agents, JFreeChart - a library for chart programming, VMFr - a framework dedicated to MAS simulation, Virtual Market - the layer of supply chains modeling in the presence of critical situation. Two upper layers create the original part of the software.

The presented model is extensively used as a simulation tool for studies of supply chains. Analyzed problems are: searching for the best configuration of chains, values of the agents' decision parameters, applied auction protocols, granularity of representation of the chains, modeling environment and its influence on the chains and, of course, forecasting and discovery of critical situations and simulation-based formation of anticrisis policies and others.

As an illustration, the authors decide to show here results dealing with one of possible critical situations which is a drop in quality of products. The drop can entail repair of the line. Alteration cost and parameters of the line are taken into account.
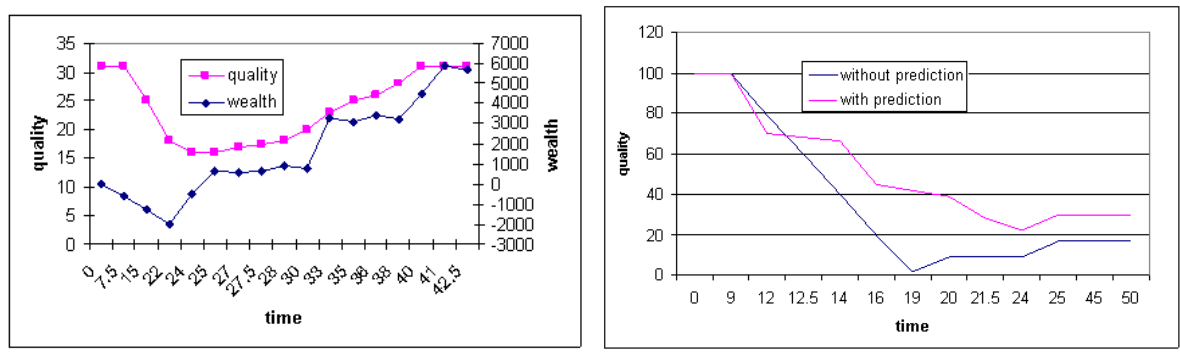

Fig. 3. a) Drop and increase of the quality of production together with changes of the capital in time b) Quality of production in time fot two strtegies: with or without prediction

The charts that group some chosen experiment results are reproduced in figure 3 Chart $a$ shows changes in time of the quality of some product under analysis. As a consequence of a line deterioration, the quality falls down successively. At some moment a repair starts and increase of the quality arises. The capital of the enterprise shows the same tendency as the quality. Initially it decreases - it is hard to sell a product of the poor quality, next things go better. 
Chart $b$ is based on the same scenario (crisis) but influence of the alteration cost is presented. Consideration of the capital allows to deepen the problem and search for an anti-crisis policy. As the alteration cost is relative high, the repair is done,,incrementally" - minimum costs just to restore the quality. Then the two strtegies are analyzed: conservative - the repair is initiated when the quality decreases beneath some assumed level, and active - with prediction of the parameters of the line.

\section{Summary}

Consideration of the article concerns with the application of the agent-based approach to the problem of management of critical situations. Design assumptions and proposal of the overall architecture of the (sub-)system dedicated to the discovery of crises and support of anti-crisis activities are described.

One of the possible applications of the presented approach can be found in a field supply chains that operate in highly dynamic and uncertain environment of economic, social, and political interdependencies that are a kind of generator of critical situations. Considerations are carried out on the basis of the originally invented model which incorporates the majority of features of supply chains.

Simulation experiments shortly presented in the article confirm the main ideas of the approach at general as well as specific levels. Future work will concentrate on further studies of the model.

\section{References}

1. Bogucki P.: A Platform for Production Planning Problems Solving Using Agent-based Approach (in polish), M.Sc., Dept. of Comp. Sci., Univ. of Science and Technology, Kraków, Poland, 2005.

2. Bussman S., Jennings R.N., Wooldridge M.: Multiagent Systems for Manufacturing Control. A Design Methodology, Springer-Verlag, 2004.

3. Dobrowolski, G., Nawarecki, E.: Crisis management via agent-based simulation. In B. DuninKeplicz, et al., editors, Monitoring, security, and rescue techniques in multiagent systems, Advances in Soft Computing, pages 551-562. Springer, 2005.

4. Kimbrough, S.O, Wu D.J., Zhong F.: Computers Play the Beer Game: Can Artificial Agents Manage Supply Chains?: Decision Support Systems 33(3): 323-333, 2002.

5. Moyaux, T., Design, Simulation And Analysis Of Collaborative Strategies In Multi-Agent Systems: The Case Of Supply Chain Management, Ph.D., L'université Laval, 2004.

6. Nawarecki, E, Koźlak, J., Dobrowolski, G, Kisiel-Dorohinicki, M.: Discovery of crises via agent-based simulation of a transportation system. In M. Pěchouček, et al., editors, MultiAgent Systems and Applications IV, volume 3690 of LNCS, pages 132-141. Springer, 2005.

7. Parunak V., Applications of Distributed Artificial Intelligence in Industry, O'Hare and Jennings, eds. in Foundations of Distributed Artificial Intelligence. Wiley 1994.

8. Swaminathan J.M., Smith S. F., Sadeh N.M., Modeling Supply Chain Dynamics: A Multiagent Approach, Decision Sciences, Volume 29, Number 3, 1998.

9. Zhao, Z., et al.: Scenario switches and state updates in an agent-based solution to constructing interactive simulation systems. In: Proc. of CNDS 2002, pages 3-10, 2002. 